\title{
Case Study on Improving L2 Writing Skills with the Contest in Higher Vocational Colleges
}

\author{
WANG Li
}

\author{
School of Humanities of Heyuan Polytechnic, Heyuan 517000 Email:angelina_190@126.com
}

\begin{abstract}
:
National English Writing Contest for technical and vocational college students is an authoritative event hosted by Higher Education Steering Committee of the Ministry of Education for ten years. Its idea and feature of test questions coincide with the orientation and teaching goal of L2 writing in higher vocational colleges. Thus, it might be an effective attempt to improve writing skills with contest and combine the contest event with daily teaching of English writing. This is to analyze case of 10 students who were tutored for writing contest. Based on the scoring criterion of the contest, the case revealed problems of L2 writing in higher vocational colleges and the significance of contest tutoring for the purpose of better monitoring teaching in the aspects of teaching contents, teachers' role and method of evaluation.
\end{abstract}

Keywords: National English Writing Contest , improving writing with contest , primary technical writing , teaching of L2 writing

\section{高职二语写作以赛促写个案研究}

\section{汪莉}

（河源职业技术学院 人文学院，广东 河源 517000)

电子邮箱:angelina_190@126.com

摘要:

全国高等职业院校英语写作大赛是教育部高教指委主办的权威性赛事。比赛已历经十年。它 的赛事理念和命题特点与高职院校二语写作定位和教学目标不谋而合。以赛促写，将大赛与 日常的写作教学结合起来，不失为一种有效的尝试。本文以竞赛指导的十位学生作为个案进 行研究, 以大赛的评分标准为依据, 试图发现高职二语写作的问题以及竞赛指导的意义, 从 而在教学内容, 教师角色, 评价方式等层面更好地指导教学。

关键词：写作大赛；以赛促写；初级技术性写作；写作教学

\section{1.引言}

随着建构主义的盛行, 二语写作教学也逐渐从简单 地注重结果产出到关注到写作的过程。趣味性生活化的 写作主题不仅能调动学生写作的积极性还能关联认知激 活写作思维, 促进创作。但是高职类院校的培养目标是 应用型技能型人才。高职类学生二语写作仅仅关注生活 化的主题是远远不够的。他们未来面对的职场环境下的 各种应用类文本的写作更多的是关注工作内容, 行业前 景, 区域特征等。高职英语写作涉及到的应用性文本按 照 Stewart ${ }^{[1]}$ 的分类标准看可以分为合作类信函 (中性信 息)、和谐类信函(正面信息)、竞争类信函(说服性信息)、 冲突类信函(负面信息)等。邀请函, 感谢信, 备忘录, 电
子邮件, 通知, 咨询信, 申请信, 失物招领, 寻物启事, 产品介绍书, 投诉信, 求职信等等功能性应用类信函都 是高职英语写作教学中的重点内容。

王传英, 王丹[2]在论及技术写作时提到就写作目的而 言技术写作属于功能性应用文体, 其目的是传递技术数 据、操作流程、使用方法或研究结论。在其论文中还展 示了 Montgomery \& Plung ${ }^{[3]}$ 从教学目的, 理论目的和专 业目的角度做出的技术写作分类图, 图中商务交际类和 技术报告类的写作都在高职二语写作教学中涉及诸多。 根据高职学生的培养目标和写作特点, 高职英语写作可 以定位为初级技术写作 ${ }^{[4]}{ }^{[5]}$ 。

全国高等职业院校英语写作大赛自 2010 年开办第一 届以来已经走过 10 年的历程, 是高职院校英语师生非常 关注的赛事之一。该大赛的举办理念和比赛内容的设置 就很好地体现了高职英语写作定位的特点 ${ }^{[5]}$ 。在大赛的 官网 ${ }^{[6]}$ 上明确写到: 大赛目的主要考查学生的英文书面 沟通、信息提取、逻辑分析、人文思辨等能力及相关素 
养, 以期通过比赛, 引领全国高职英语写作教学的改革 与发展, 促进学生英语应用能力的整体提高, 为其未来 职场发展和个人可持续发展奠定良好的基础。大赛的初 赛和复赛的题型分为两部分: 第一部分就是常见的职场 背景下的应用文技术写作, 字数不少于 200 词; 第二部 分为图表作文或图画作文。字数不少于 300 词。从赛题 的命题特点来看, 一方面是学生初级技术写作能力, 考 查职场环境下的书面交际能力, 另一方面是技术分析能 力, 考查对各类图表图画的解读分析和洞察能力。从它 的目的和命题特点来看, 它与高职人才培养定位以及英 语写作教学目标是不谋而合的。因此, 以赛促写不失为 一种激发学生动机, 提高高职学生写作水平的重要手段。

\section{2. 研究设计}

以赛促写的成效需要老师和学生共同的努力才能实 现。那么, 在指导学生参赛过程中, 笔者对参赛的学生 进行了个案研究, 以期能够对未来的教学和竞赛指导带 来一定的启示。

\section{1. 研究问题}

本研究着重调查了教师的培训过程和学生的写作练 习过程, 尝试回答下面两个问题:

(1) 高职学生二语写作问题体现在哪里?

(2) 竞赛指导在多大程度上提升写作水平?

2. 研究对象

此次研究的对象为参加高等职业院校写作大赛初赛 笁选出来的十名学生。这十名学生通过教师一定时间的 写作指导, 由教师再次笁选推荐参加省赛, 并取得较好 的成绩。

3. 研究工具

本研究使用了访谈和写作文稿批改。访谈分为辅导 前的访谈和辅导结束后的访谈, 共两次。了解学生对自 身写作水平的认知和竞赛指导对提升或改进写作的作用 等。同时通过批改写作文稿, 总结学生二语写作出现的 问题，检验竞赛指导对提升写作水平的影响程度。

\section{3. 研究过程}

教师在对这 10 名学生进行为期十周的培训每周布置 一篇作文。数据收集过程包括了对访谈的录音整理和 10 名学生共 100 篇文稿的批阅和统计。

教师指导方式具体表现在: 每周有布置阅读和写作 任务。写作的内容根据大赛的样题出, 主要为应用文写 作和图表作文写作。学生随时与老师进行沟通。教师检 查学生的给定背诵内容, 以及学生的 reading notes 和 summary. 除此之外, 教师每周集中一次作文讲解与分析。 教师提前收到学生发的作文电子稿并批阅, 作文分析讲 解过程中, 同学们会带上纸质稿作文相互讨论修改, 发 现问题, 教师点评并反馈信息, 课后学生对自己的习作

\section{4. 结果分析}

\section{1. 学生的习作分析}

学生写作文稿按照大赛的评分标准进行评定, 即信 息提取、分析与整合 (25\%), 内容与逻辑 $(35 \%)$, 语法、 词汇和语域 $(30 \%)$, 格式与字数 $(10 \%)$ 。不仅考查学生 格式和语言使用的准确性, 还考查学生对信息提取的能 力以及内容和逻辑的完整性。

\section{表一 十名学生作文错误分布点及总错误占比}

\begin{tabular}{l|r|r|r|r|r|r}
\hline \multirow{2}{*}{ 作文评分点 } & \multicolumn{2}{|c|}{ 第一次作业 } & \multicolumn{2}{|c|}{ 第十次作业 } & \multicolumn{2}{c}{ 其他八次作业 } \\
\cline { 2 - 7 } & 错误数 & 占比 & 错误数 & 占比 & 错误数均值占比 \\
\hline 信息提取、分析与整合 & 8 & $12.1 \%$ & 4 & $8.7 \%$ & 4.4 & $7.7 \%$ \\
\hline 内容与逻辑 & 5 & $7.6 \%$ & 5 & $10.9 \%$ & 8.9 & $15.6 \%$ \\
\hline 语法、词汇和语域 & 45 & $68.2 \%$ & 34 & $73.9 \%$ & 39 & $68.2 \%$ \\
\hline 格式与字数 & 8 & $12.1 \%$ & 3 & $6.5 \%$ & 4.9 & $8.6 \%$ \\
\hline 总计 & 66 & $100.0 \%$ & 46 & $100.0 \%$ & 57.2 & $100.0 \%$ \\
\hline
\end{tabular}

上面表一列出了 10 名学生的作文错误点分布和占比 情况。从表中可以看出, 学生的第一次作文中在信息的 提取分析以及格式上的错误非常多, 10 人当中就有 8 人 都出现这两处的问题。在最后一次作文中这两处得到了 明显的改善, 中间的八次作文也能看出学生的循序渐进。 另外从总体的错误数也由 66 处降到 46 处, 学生整体上 水平有所提高。不过, 就内容与逻辑这一点来看, 学生 并没有得到明显的提高。另外，无论从哪一次作文错误 数和占比都可以看出, 语法词汇等方面的错误数总是最 多的。这一点符合他们高职英语普遍基础薄弱的特点。 即使是像这些相对比较好一点的同学, 作文当中的这部 分的错误仍然平均有 3 处左右。

首次写作，学生还没有接受一定量的指导和培训, 对很多作文的格式比较陌生, 也不清楚该如何对作文题 干中出现的内容信息进行提取分析。作文 1 是销售经理 针对客户的投诉问题要安排和总经理开会讨论三点信 息, 结果是大部分学生将开会的对象定为对方公司人员, 这就是信息提取错误。这种信息提取和分析问题不仅在 应用文题干中出现, 更多的是体现在对图表作文的图表 分析上。在经过教师的培训指导, 学生了解了相关的表 达和分析方法, 并在归纳这些信息上有了一定的信心。

在内容和逻辑上, 学生出现的问题一是内在的逻辑 性不强, 结构不清晰。有些人为了让文章看起来比较条 理清晰, 就特别爱用 First, Second 等词, 往往使文章读起 来更生硬，句间关联性很怪异。如某同学写到“First of all, I really want to have a meeting with....Second, ...hope you will give us a chance to have a meeting....Third, I hold my view that we should have a discussion about....”这三点本 质上是一个内容, 却生硬地用词套, 加上穴余重复信息, 让文章变成硬凑成的“八股文”的味道。“...In2000, google began to sell advertising on their website....Through this chart, we know google is one of the greatest examples of online success. ”这篇作文在讲解图表所呈现的内容之后, 
用文的格式要求，并有了很大的提升。

2. 指导老师的竞赛辅导

在为期十周的培训中, 教师一方面以阅读来增加学 生的输入量, 另一方面以写作来检验他们的输出效果。 Swain ${ }^{[7]}$ 提出的可理解输出假设认为, 语言的输出有三大 功能。第一种功能(注意或触发功能)是指在语言输出过程 中学习者会注意到自身表达上的差距; 第二种功能(假设 检验功能)是学习者在目标语输出过程中不断假设和修改 假设的检验过程; 第三种功能(元语言反思功能)是用语言 来解释语言, 提高语言反思能力的功能。这三大功能对 提高二语表达的准确性和流利性有很大作用。教师在竞 赛辅导过程中, 通过向学生传达大赛的写作题型的要求 和初级技术性写作的特点, 引导学生将语言的注意功能 发挥在技术性写作的选词造句和语言点上, 将假设检验 功能运用在培训辅导过程中的文本创作以及对文本的师 生反馈和自我修正上, 将元语言反思功能体现在多稿修 改过程中的语篇重构、多样化的句法表述上。

竞赛辅导的过程中, 教师指导学生进行了若干类 型题材的写作, 从结果来看, 发现学生图表作文撰写能 力差, 具体体现为分析能力有限, 整体思路不够清晰。 另外, 学生自我纠错能力不足, 但在讨论和同伴互改中 却常常能觉察出别人的问题所在。教师针对学生的这些 问题, 同时结合竞赛的作文特征进行分门别类的指导。 总体上讲, 培训的效果不太显著但均有所提升。根据大 赛的评分标准, 教师跟踪了每位学生的写作作品, 进行 了针对性的批改, 对各次批改结果进行了比对, 以期能 结合大赛的要求促进日常教学工作。

下图为学生十次初稿的成绩。

最后在格式上往往是不完整或位置错误，但经过培 训和阅读大量的应用文, 学生有意识地注意到了不同应

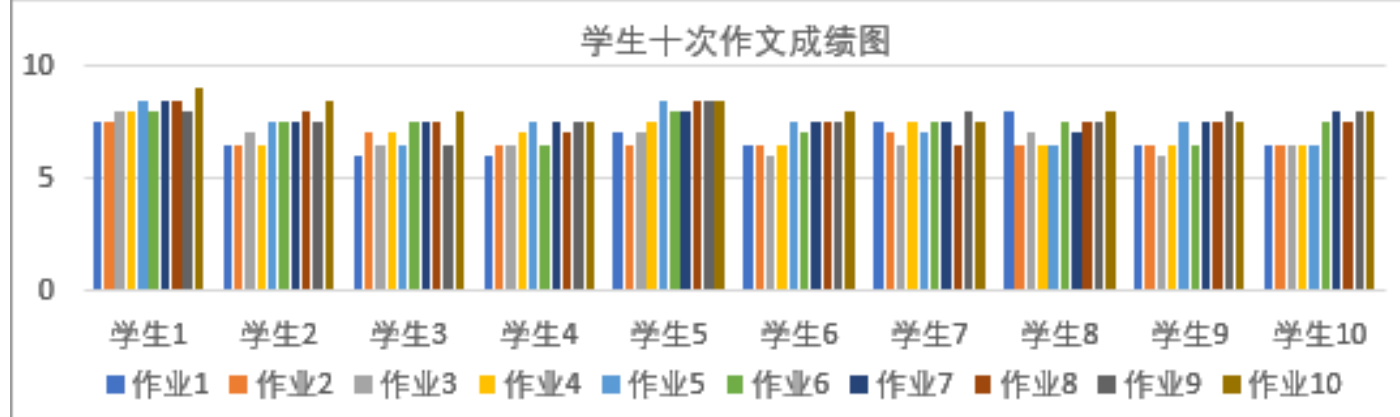

相对于课堂教学, 以竞赛为切入点结合教师辅导能 带给学生: 1) 积极的竞赛意识, 这是在学习动机上对学 生产生的正面影响; 2) 输入输出量的增加, 这是培训辅 导的强化特色; 3) 师生交互性增强, 教师的讲解、点评 和同伴互评都比平时的课堂教学更有效果。这些因素是 教师竞赛辅导的积极意义所在。

3. 学生访谈分析

针对学生的竞赛热情和对自身写作的认知, 培训前 的访谈中发现, 学生对于竞赛写作的要求和认知不足。 90\%的学生不知道技术性写作, 不会或者未接触到图表作 文。培训结束后学生都表示掌握了很多技术性写作的方 法和技巧, 对图表作文也有了一定的认识。
关于期待怎样的一种辅导培训, 他们大部分同学还 是希望老师能多讲解范文, 总结句型, 指出错误和改正 方法等, 这也体现了学生的传统意识很强, 对老师有过 分的依赖。培训结束后 $90 \%$ 的学生认为教师做到了这些。 在谈到对培训哪些环节比较喜欢时, $80 \%$ 的人对学生互评 反馈比较喜欢。说明他们的学习意识上已经发生了一些 改变, 由被动接受, 到主动思考和分析。李同学谈到“...... 之前很多问题没有意识到, 后来 (同伴) 互评, 让我一 下子明白过来自己错在哪里, 为什么错了, 当时的印象 非常深刻。”

在问及有效的提高写作的方法时, 听说读写都有同 学提到, 但说得多的还是阅读和背单词。说明大家对输 
避免单一评价给老师带来巨大的压力，给学生成绩带来 无效甚至消极的影响。

的自我水平评价中, $50 \%$ 人认为自我写作水平有所提高。 张同学说到“一直不太喜欢阅读, 看不太懂, 好多单词不 认识。(老师)您一直有让我们看书, 安排阅读的内容, 只 好硬着头皮看下去, 结果, 坚持一段时间觉得阅读和写 作都没那么难了, 也不知道是不是阅读真的起了作 用。…...”

最后在谈到收获时, 值得欣慰的是每一位同学都认 为他们对写作产生了更浓厚的兴趣。Izumi ${ }^{[8]}$ 认为人在说 话或者是写作时, 大脑内部存在一个编辑、修正、监控 系统, 通过这个系统, 学习者可以发现自己在语言表达 方面的不足, 或曰“语言缺失”, 从而激发他的学习欲望, 并寻求渠道进行修补。教师的辅导和写作训练在一定程 度起到激发学生的学习兴趣和欲望的作用。但是值得一 提的是, 仍然有个别同学觉得没有什么特别大的提高, 这可能跟学生的学习时间安排, 培训跟进, 以及教师自 身培训内容和方法有关。教师应总结和反思培训方式、 材料和内容等, 争取在提升培训更大成效方面进行改进。

\section{5. 讨论}

学生的写作反映出了很多的问题。学生对初级技术 性写作中信息甄别和内容分析不足, 词汇语法问题仍为 突出。教师的竞赛辅导在一定程度上有助于提升学生的 写作成绩。在访谈中也能感受到竞赛辅导给学生带来的 写作认知上和心理上的变化。那么将这些发现运用到日 常教学中, 我们应该做到以下几个方面。

\section{1. 在教学内容上做到以赛促写}

首先应加强初级技术性写作意识, 结合实际的商务 工作场景, 锻炼学生恰当的书面沟通和交际能力; 其次 应加大文本输入输出量。平时教学中, 学生大部分时间 都关注在课本内的阅读和听力上, 课下的阅读和听力输 入极其有限。教师可以布置相关的内容, 将任务带入课 堂, 引导和监督学生参与并鼓励文本写作, 逐渐摆脱中 式英语, 提升遣词造句的能力; 最后还应该引导学生对 社会热点问题进行深入的观察和分析, 因为图表作文中 的话题和数据都往往是基于社会各方面的事件。学生解 读信息, 分析和解决问题的能力不仅仅是二语写作中的 一个考验, 更是将来面向社会的一种生存技能。

2. 在教师角色上做到多元化

教师应更多的是一个组织者, 观察者和协助者。竞 赛辅导环境下学生能感受到更多的关注, 积极性和认真 度也能随之提高。但平时教学中, 教师面对众多的学生 很难做到对学生最大化的关注。这种情景下, 我们教师 就应该幻化成多元的角色, 利用信息技术和线上微课给 学生带来“一对一”式的互动式体验学习。组织学生线上自 主学习, 观察学生的线上学习数据, 并给予反馈, 同时, 当学生遇到学习困难时, 协助解决, 达到促学的目的。

3. 在评价方式上做到多样化

作文人工的批改工作量巨大, 教师可适当结合批改 网站实现自动批改。同时, 在课堂上可选取有代表性的 作文进行集中点评, 学生也可以参与互评等, 总之, 应

\section{6.结语}

初级技术性写作是高职学生二语写作的实质, 它与 未来的岗位能力需求紧密相连。高职院校英语写作大赛 为学生提高写作注入强心剂。通过竞赛辅导, 学生不仅 对写作有了更大的兴趣和进步, 更是对未来的职场应对 和语用交际能力产生信心。从个案研究中可以看出, 以 赛促写从客观上能起到一定的作用。那么在常规化课堂 息化教学手段将竞赛辅导的优势惠及到更大的学生群 体, 同时在反馈评价方式上更有效地激发学生的学习潜 能, 这样的做法不失为值得一试的尝试。

\section{作者简介:}

姓名 (1981 -), 女, 安徽池州人, 讲师, 硕士, 主 要从事应用语言学及二语写作研究。邮箱: angelina_190@126.com

\section{REFERENCES}

[1]Stewart, M., K.Zimmer\&L Clark.Business English and Communication[M].New York:McGraw Hill, 1984

[2]Wang Chuanying, Wang Dan. Technical Writing and Training of Professional Translators[J]. Journal of PLA University of Foreign Languages, 2011, (2) :69. illustrated taxonomy of technical writing [C]. Professional Communication Conference. Seattle: IPCC'88 Conference Record, 1988: 141-146.

[4]Liu Dailing. Comprehensively Promote the Reformation of Higher Vocational College English With Unity, Cooperation, Pragmatism and Innovation[C]. Kunming: 2009 Working Conference Of Directors And General Secretaries Of Higher Vocational College Professional Teaching Steering Committee for Ministry Of Education, 2009

[5]Jiang Han. Effects of English Writing Contests to the Development of Vocational Writing Curriculum at Polytechnics[J]. Journal of Guangzhou Panyu Polytechnic, 2011, 6:25.

[6] Official website of National English Writing Contest for technical and vocational college students. Agenda and Rules[EB/OL][2019-12-10]. http://www.writing-contest.cn/2015/about/419828.shtml. [7] Swain M. Three functions of output in Second 教学中将竞赛的理念和内容融入到教学任务中, 利用信

[3]Montgomery, T. T. \& D. L. Plung. A definition and 
[8] Izumi S. Comprehension and Production Processes in

Language Learning [A]. In G. Cook \& B. Seidlhofer (eds). Principles and Practice in Applied Linguistics: Studies in Honour of H. G. Widdowson[C]. Oxford: Oxford University Press, 1995:125-144
Second Language Learning: In Search of the Psycholinguistic Rationale of the Output Hypothesis[J]. Applied Linguistics, 2003, 24(2):168-196. 